\title{
Acute Care Surgery: A Necessity Across Europe. Are We Ready to Take the Lead?
}

\author{
Hayato Kurihara \\ Department of Surgery, Emergency Surgery and Trauma Unit, Humanitas Research Hospital, Milan, Italy
}

\author{
Corresponding author: \\ Hayato Kurihara, MD, FEBSEmSurg \\ Head of Emergency Surgery and \\ Trauma Unit, Department of Surgery \\ Humanitas Research Hospital \\ Via Manzoni 56, 20089 Rozzano, \\ Milan, Italy \\ E-mail: hayato.kurihara@humanitas.it
}

Received: 21.08 .2017

Accepted: 30.08 .2017
In 2008 Uranues published the results of a questionnaire sent to experts across 27 European countries on the assessment of attitudes toward acute care surgery and he concluded that no unified system of acute care surgery in Europe was yet developed due to different approaches to the surgical critical patient and that, with exception of some dedicated centres, the intra-hospital resources were not dedicated to acute care surgery patients with consequent negative impact on ideal treatment both in the elective and acute patients (1).

The problem in most of European countries is still the same: who is the best surgeon to deal with these patients with an acute surgical condition? The era of the "omnipotent general surgeon" is to an end and in order to improve quality in specific areas many institutions dedicate most of their economic resources to highly specialized surgical units; nowadays young committed surgeons are therefore attracted by the so-called "organ specific surgery" and take care of emergency surgery and trauma cases just because of a contractual obligation.

The lack of subspecialty in trauma and acute care and the lack of interest in the treatment of such complex surgical and trauma cases did not give impetus to the development of clinical standards or professional accreditations guidelines causing a perfect storm with consequent provided care that is less than optimal.

Furthermore, we are living in an aging society with patients that are progressively frailer with less physiologic reserves. Emergency surgery should not be considered just a form of elective surgery performed out of hours and adequate care should focus not only on anatomy and pathology, but also and 
particularly on patient's physiology and rearrangement of impaired physiology should be one of the essential key of the care of such patients.

For years standard training in surgery has been clearly centred on anatomy, pathology and technical issues, and surgeons progressively showed a lack of interest in critical care aspects leaving to anaesthesiologists and intensivists the hard task to correct physiology impairment; the so call closed ICU system, although reasonable especially in terms of redistribution of staff resources, had some impact in term of better outcomes, but also led surgeons to spend less time in ICU itself however, when facing such patients we should spend more time with our colleagues in ICU being more proactive in the decision-making process.

As in trauma, many European countries did not develop yet and effective trauma system and have no national trauma registry; therefore, they are far away to obtain an adequate benchmark of levels of trauma care. Acute care surgery issues are very similar to trauma care and quality improvement programs should also be set up through specific clinical and organizational pathways and probably through acute care registries.

As every year, during the last Congress of the European Society of Trauma and Emergency Surgery - E.S.T.E.S. (2) recently and successfully organized by Professor Mircea in Bucharest in May 2017, many distinguished surgeons from all over the world had the opportunity to gather together proposing new ideas and inspiring new projects one to another, but this is unfortunately not enough since we need a specific curriculum for trauma and emergency surgery with extended focus on patients' needs.

Thank to E.S.T.E.S. and the European Union of Medical Specialists (U.E.M.S. Union Européene des Médicine Spècialists) (3), less than 10 years after Prof. Uranues survey, a specific curriculum to become Fellow of the European Board of Surgery Emergency Surgery has been developed and from 2016 some surgeons started to can be certified.

The curriculum is specifically designed for these surgical and critically ill patients and focuses on different peculiar aspects such as bleeding, inflammation, infection, obstruction, ischemia, compartment syndrome and organ dysfunction, but there are other aspects that an acute care surgeon should consider: leadership and teamwork ability. These two aspects, that should be indeed part of any surgeon, in stressful situations where time matters and swift decision making is crucial, might make the difference in patient outcome.

The present generation of surgeons has a huge responsibility toward their residents in surgery since and adequate balance between the need of highly specialized surgical competences and optimal patient management with a "physiology oriented decision making" is needed.

\section{References}

1. Uranues S, Lamont E. Acute care surgery: the European model. World J Surg. 2008;32(8):1605-12. doi: 10.1007/s00268-0089501-4.

2. http://www.estesonline.org

3. https://www.uemssurg.org/divisions/emergency-surgery 